\title{
Assessment of PIT tag retention and post-tagging survival in metamorphosing juvenile sea lamprey
}

\author{
Lee G. Simard ${ }^{1}$, V. Alex Sotola ${ }^{1}$, J. Ellen Marsden ${ }^{1}$ and Scott Miehls ${ }^{2^{*}}$ (I)
}

\begin{abstract}
Background: Passive integrated transponder (PIT) tags have been used to document and monitor the movement, behavior, or survival of numerous species of fishes. Data on short- and long-term survival and tag retention are needed before initiating studies using PIT tags on a new species or life stage. We evaluated the survival and tag retention of 153 metamorphosing juvenile sea lamprey Petromyzon marinus tagged with 12-mm PIT tags on three occasions using a simple surgical procedure.

Results: Tag retention was 100 and $98.6 \%$ at $24 \mathrm{~h}$ and $28-105$ days post-tagging. Of the lamprey that retained their tags, $87.3 \%$ had incisions sufficiently healed to prevent further loss. Survival was 100 and $92.7 \%$ at $24 \mathrm{~h}$ and $41-118$ day post-tagging with no significant difference in survival between tagged and untagged control lamprey. Of the 11 lamprey that died, four had symptoms that indicated their death was directly related to tagging. Survival was positively correlated with sea lamprey length.
\end{abstract}

Conclusions: Given the overall high level of survival and tag retention in this study, future studies can utilize 12-mm PIT tags to monitor metamorphosing juvenile sea lamprey movement and migration patterns.

Keywords: Lamprey, PIT, Retention, Tag effects, Survival

\section{Background}

Sea lamprey (Petromyzon marinus) have had dramatic negative impacts on fish populations in the Great Lakes $[1,2]$. Beginning in the 1950s, state, provincial, and federal agencies have worked to suppress sea lamprey populations to facilitate restoration of native Lake Trout (Salvelinus namaycush) populations. Effective, targeted control of nuisance species requires an understanding of the species' life history and movements. Sea lamprey are a semelparous, adfluvial species; after hatching in spring, larval sea lamprey (ammocoetes) spend 4-6 years buried in stream sediments before they metamorphose into parasites, migrate downstream, and spend 12-18 months in lakes before migrating back into streams to spawn.

\footnotetext{
${ }^{*}$ Correspondence: smiehls@usgs.gov

2 U.S. Geological Survey, Great Lakes Science Center, Hammond Bay

Biological Station, 11188 Ray Road, Millersburg, MI 49759, USA

Full list of author information is available at the end of the article
}

Control efforts, including selective lampricides and barriers to block migrations, focus on the larval and spawning phases, as the parasitic juveniles are too dispersed to target $[2,3]$.

Continuing issues with permitting and nontarget mortality associated with use of lampricides and stream barriers have motivated a search for alternative control methods. Alternative methods such as male sterilization and trapping with pheromone attractants have been developed to target spawning adults and limit reproductive success $[4,5]$. The life stage that has received the least attention for control is the outmigrating juvenile stage $[1,2]$. As with spawning adults, the migratory behavior of this life stage makes them vulnerable to capture at a single in-stream location. Metamorphosing juvenile sea lamprey were targeted in the 1940s and 1950s using dams and inclined screen traps [2, 6, 7], but these efforts were discontinued following the discovery of selective chemical lampricides and limited research on this life stage has 
occurred since then. A better understanding of the spatial and temporal patterns of outmigration is needed to develop efficient methods to target this life stage.

PIT tags have been used to track in-stream movement and migration patterns of many fish species [8-10]. PIT tags are small, individually coded capsules that range from 8 to $32 \mathrm{~mm}$ in length. Tags are implanted in individual fish and can be read with a handheld scanner or remotely when the fish passes an electromagnetically charged antenna [11, 12]. Antennas can be designed to encompass cross sections of a stream and detect tagged individuals that pass the antenna array without physically recapturing the fish $[13,14]$. Antennas can be constructed to withstand high discharge events and periods of high debris loading that would be likely to dislodge or destroy other sampling gear $[12,15,16]$. These characteristics make PIT antenna systems ideal for monitoring juvenile sea lamprey outmigrating during the fall and spring.

When using implanted tags to track movement or behavior of individual fish, it is important to ensure tags are retained and do not affect survival or behavior. In prior studies, sea lamprey ammocoetes [17] and juvenile Pacific Lamprey (Lampetra tridentata) $[18,19]$ had high survival and tag retention when tagged and held at temperatures between 8 and $23{ }^{\circ} \mathrm{C}$. However, the tagging procedures in these studies required dissecting microscopes, sutures, parafilm bandages, and multiple needles and lacked the simplicity needed for large-scale field deployment. In response, a less-invasive procedure [20] was tested on juvenile Pacific Lamprey using a single incision, a 9-mm FDX PIT tag inserted by hand, and no sutures or bandages. Lamprey were held between 9 and $18{ }^{\circ} \mathrm{C}$ and showed similarly high survival and tag retention as the studies with more complicated procedures [20].

No prior studies have been conducted with tagged metamorphosing juvenile sea lamprey or have tagged and held juveniles under natural conditions. Although lamprey survival tends to increase when held at lower water temperatures $[19,20]$, survival has not been tested at temperatures as low as the $5{ }^{\circ} \mathrm{C}$ which is thought to be the upper threshold for inducing outmigration by juvenile sea lamprey [6]. Additionally, survival and tag retention has not been tested for juvenile lampreys tagged using the simple procedure with the larger 12-mm HDX PIT tags [12]. We tested the survival and tag retention of metamorphosing juvenile sea lamprey implanted with 12-mm HDX PIT tags using the less-invasive tagging procedure $[20]$ and held at water temperatures that would be experienced during their natural outmigration, to (1) determine whether tagging affected short- and long-term survival and (2) quantify tag retention for juvenile sea lamprey.

\section{Methods}

\section{Lamprey collections}

Metamorphosing juvenile sea lamprey were captured in the Fort River, MA, during September 23-27, 2013, from stream sediment using backpack electrofishers (Model ABP-2, ETS Electrofishing Solutions, Madison WI) following US Fish and Wildlife Service standard collection protocol (slow pulse $=3 \mathrm{pps}$; fast pulse $=30 \mathrm{pps}$; duty cycle $=25 \%$; burst $=3.1$; voltage $=125$; [21]). Sea lamprey were temporarily held at the Conte Anadromous Fish Research Laboratory, Turners Falls, MA, in $2.0 \mathrm{~m}$ diameter $\times 1.5 \mathrm{~m}$ depth $(4000 \mathrm{~L})$ circular flowthrough tanks fed with water from the Connecticut River, with $10-15 \mathrm{~cm}$ deep river sediment covering the bottom. Fort River sea lamprey were transported to the Rubenstein Ecosystem Science Laboratory on October 10, 2013. Juvenile sea lamprey were also captured during outmigration from two tributaries of Lake Champlain: Morpion Stream, QC, Canada, and Mallets Creek, Colchester, VT, between October 28 and December 2, 2013; these lamprey were held in-stream in cylindrical mesh cages $(29 \mathrm{~cm} \times 42 \mathrm{~cm})$ and transported to the Rubenstein Laboratory on December 6, 2013. Fort River and Lake Champlain sea lamprey were held in separate tanks.

\section{Experimental tanks}

Sea lamprey were housed in either round (observation) tanks $(1.2 \mathrm{~m} \times 0.8 \mathrm{~m}$ polyethylene) or a rectangular (holding) tank $(0.42 \mathrm{~m} \times 0.66 \mathrm{~m} \times 0.28 \mathrm{~m}$ plastic). All tanks were fed with recirculating dechlorinated water from a single $568-\mathrm{L}$ head tank with a $1 / 8 \mathrm{hp}(93.3 \mathrm{~W})$ pump at a rate of approximately $20 \mathrm{~L}$ per minute. Water temperature was maintained between 3.7 and $6.5^{\circ} \mathrm{C}$ for the duration of the study except two events during which water was warmed to $>10{ }^{\circ} \mathrm{C}$ (February 13-14 and February 24-26) and then rapidly cooled to $<5{ }^{\circ} \mathrm{C}$ during a 24-h period. Water temperature was maintained using a 1-hp (746 W) inline chiller (Delta Star, Aqua-Logic, Inc.). Beach sand $2-10 \mathrm{~cm}$ deep covered the bottom of all tanks. A simulated fall photoperiod with $11 \mathrm{~h}$ of light and $13 \mathrm{~h}$ of darkness was maintained using overhead fluorescent lights set on a timer. The rectangular holding tank was maintained at $0.22 \mathrm{~m}$ water depth $(74 \mathrm{~L}$ capacity). The circular tanks were maintained at either 0.42 or $0.76 \mathrm{~m}$ water depth (340 or $620 \mathrm{~L}$ capacity, respectively). Counterclockwise flow was maintained at a rate of 0.05$0.15 \mathrm{~m} / \mathrm{s}$, measured with a portable, Marsh-McBirney flow meter (model 201D), around a center column placed in both circular tanks using $38-\mathrm{L} / \mathrm{min}$ pumps. Water depth, temperature, and circulation velocity were varied in the two circular tanks as part of a separate behavioral observation study. 


\section{Tagging procedure}

Lamprey were anesthetized by immersion in a $0.026 \mathrm{~mL} / \mathrm{L}$ concentration of AQUI-S 20E (AQUI-S, New Zealand). When lamprey became unresponsive, they were removed from the anesthetic, weighed to the nearest $0.01 \mathrm{~g}$, and measured to the nearest $1 \mathrm{~mm}$. A sterile scalpel was used to make a $2-3-\mathrm{mm}$ incision on the left lateral side approximately $20 \mathrm{~mm}$ posterior to the gill pores. A half-duplex PIT tag $(12 \mathrm{~mm} \times 2.12 \mathrm{~mm}$, $83 \mathrm{mg}$, Oregon RFID) was inserted by hand and guided posteriorly into the body cavity away from the incision. Implanted tags have been observed to move posteriorly during healing [20]; therefore, we inserted tags posteriorly through the incision to prevent potential reopening of the wound and tag shedding as the tag moved past the incision. Tagged lamprey were placed in an aerated bucket with fresh water until they became mobile and then transferred to a 38-L observation tank set in a chilled water bath (held at same temperature as the larger holding tanks) for $24 \mathrm{~h}$ to monitor short-term survival and tag retention. Lamprey were then stocked into a 1.2-m circular tank described above. Untagged control lamprey were measured after the observation period, to avoid stress related to handling; controls were a random subset of the lamprey from each site and therefore assumed to have a similar length range to the tagged lamprey in their respective groups.

\section{Short-term survival after tagging}

Two groups of sea lamprey from the Fort River were used for direct comparison of survival for tagged and untagged (control) animals. Group 1 sea lamprey $(n=20$ tagged; 23 control) were tagged November 19, 2013, and group 2 sea lamprey ( $n=20$ tagged; 15 control) were tagged December 6, 2013; each group was housed in a separate observation tank. Tanks were monitored daily Monday to Friday, and sea lamprey were removed if dead, or generally unresponsive and showing obvious signs of impairment (i.e. minimal reaction to stimulus, large protruding viscera, etc.), or exhibiting signs of fungal growth. Any visible symptoms were recorded at the time of removal. The effect of tagging on survival was evaluated by comparing Kaplan-Meier survival curves of tagged and control sea lamprey from groups 1 and 2 at 24 h post-tagging and on January 22, 2014, 64 day (group 1) and 47 day (group 2) post-tagging using a log-rank analysis.

\section{Long-term survival and tag retention}

After conclusion of the tagged versus control observation period, all tagged sea lamprey from groups 1 and 2 were stocked into a common observation tank and held for long-term assessment. Additionally, 110 sea lamprey from Lake Champlain tributaries, hereafter referred to as group 3, were tagged February 4, 2014, and stocked into a separate observation tank; for these lamprey, survival during the 60 day prior to tagging was monitored for comparison with tagging mortality. The long-term survival of all tagged sea lamprey was monitored until March 17, 2014.

Initial total length of sea lamprey was compared among tagging groups using ANOVA, and length differences between groups were identified using Tukey-Kramer HSD test. A logistic regression analysis using a likelihood ratio test (LRT) was used to determine whether total length influenced survival. All analyses were conducted in JMP ${ }^{\circledR}$ Pro 11 (SAS Institute Inc., Cary, NC).

All tagged sea lamprey were scanned for tags using a PIT reader, and incisions were inspected on March 4, 2014. Percent tag retention was calculated for each tagging group. The proportion of sea lamprey in each tagging group with wounds sufficiently healed to no longer be vulnerable to tag loss was calculated. Incisions with healed skin, healed muscle, and the internal muscle appearing closed were considered sufficiently healed to prevent tag loss while incisions that remained open or with bulging muscle were considered still vulnerable to tag loss.

\section{Results}

\section{General observations}

A total of 153 sea lamprey with a mean initial length of $162 \mathrm{~mm}$ (range 129-205 mm; Fig. 1; Table 1) were tagged as part of three groups. Analysis of variance indicated significant variation in length among tagged lamprey from the three tagging groups $(d f=2, F=44.24$, $p<0.0001)$. There were no significant differences in length between group 1 (147.4 mm, range 129-174) and group 2 (mean $=148.0 \mathrm{~mm}$, range $135-168)$ tagged Fort River lamprey ( $p=0.98)$; however, tagged group 3 lamprey from Lake Champlain lamprey were significantly larger $($ mean $=167.8 \mathrm{~mm}$, range $129-205)$ than both Fort River groups $(p<0.0001)$.

Three tagged lamprey from group 3 died by becoming impinged on a pump intake, entangled in netting, or attacked by another lamprey; these lamprey were excluded from the overall survival calculation. Four tagged lamprey died with viscera protruding from the incision 5-17 day after tagging, a symptom that can be directly attributed to the tagging process (Table 2). Two additional tagged lamprey died with small bulges at their incision; however, it was not uncommon to see other lamprey with similar bulges that survived. During the 3 weeks post-tagging, four tagged lamprey died with no external symptoms and four untagged lamprey died without external symptoms. One tagged and three untagged lamprey had fungus along their tails and were removed prior to death to avoid spreading the fungus to other lamprey. 


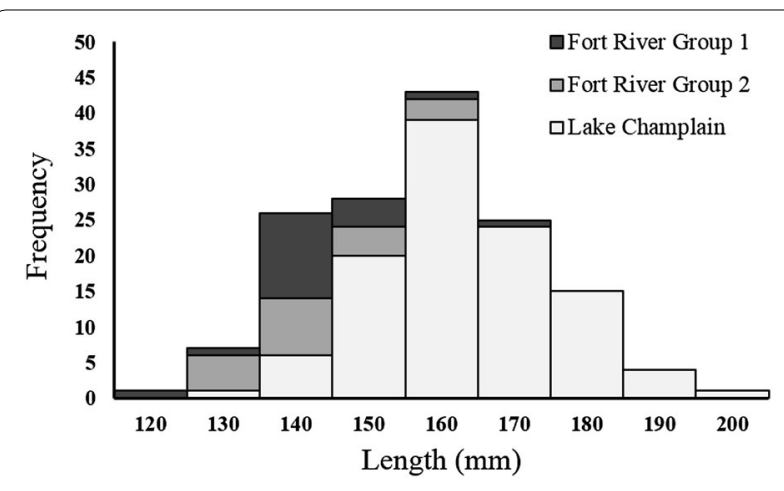

Fig. 1 Length frequency of metamorphosing juvenile sea lamprey collected from the Fort River, MA and two tributaries to Lake Champlain used to assess survival after implantation with 12-mm HDX PIT tags. Fort River sea lamprey were divided into two groups for survival comparison between tagged and untagged and held separately from each other as well as Lake Champlain sea lamprey

\section{Survival}

Survival of all tagged sea lamprey for all three groups $24 \mathrm{~h}$ post-tagging was $100 \%$ (Table 1 ). During direct comparison of tagged to control animals, survival of tagged sea lamprey in groups 1 and 2 was 90\% (18 of 20 ) at 64 day post-tagging and $85 \%$ (17 of 20) at 47 day post-tagging, respectively (Table 1; Fig. 2). Survival of all untagged sea lamprey in groups 1 and 2 was 100\% (38 of 38 ) for the duration of the observation periods (64 and $47 \mathrm{~d}$, respectively). There was no significant difference in survival between tagged and untagged lamprey in group 1 (log-rank test: $\chi^{2}=2.360, D F=1, p=0.1245$ ) or group 2 (log-rank test: $\chi^{2}=2.372, D F=1, p=0.1235$ ). Tagged sea lamprey from groups 1 and 2 were held for observation for an additional 54 day during which no additional mortality was observed (Fig. 2). Survival of group 3 sea
Table 2 Symptoms of deceased metamorphosing juvenile sea lamprey from both tagged and untagged groups used as part of a survival analysis study

\begin{tabular}{llll}
\hline $\begin{array}{l}\text { Symptoms } \\
\text { observed }\end{array}$ & $\begin{array}{l}\text { Number } \\
\text { of mortalities }\end{array}$ & $\begin{array}{l}\text { Median days } \\
\text { until death }\end{array}$ & $\begin{array}{l}\text { Maximum days } \\
\text { until death }\end{array}$ \\
\hline $\begin{array}{l}\text { Tagged lampreys } \\
\begin{array}{l}\text { Protruding } \\
\quad \text { viscera }\end{array}\end{array}$ & $4(2.6)$ & 8 & 17 \\
No symptoms & $4(2.6)$ & 41 & 45 \\
$\begin{array}{l}\text { Small bulge } \\
\text { Fungus }\end{array}$ & $2(1.3)$ & 35 & 35 \\
$\begin{array}{l}\text { Untagged lampreys } \\
\text { No symptoms }\end{array}$ & $4(3.3)$ & 27 & 27 \\
Fungus & $3(2.5)$ & 19 & 23 \\
\hline
\end{tabular}

The number of mortalities observed for each symptom type, median, and maximum number of days until death for lampreys with each symptom are shown; percentage of mortalities among all tagged and untagged lamprey is given in parentheses

lamprey prior to being tagged was $94.2 \%$ (113 of 120; 60 day observation period) and $94.5 \%$ (104 of $110 ; 41$ day observation period) post-tagging. Overall survival for all tagged sea lamprey at the end of the study was 92.7\% (139 of 150; Table 1). Survival of tagged lamprey at the end of the experiment was significantly related to total length $\left(\chi^{2}=4.01, D F=1, p=0.0452\right)$, with longer lamprey exhibiting higher survival.

\section{Tag retention}

Tag retention was $100 \% 24 \mathrm{~h}$ post-tagging (Table 1 ) for all tagging groups. Of the surviving lamprey evaluated on March 4, 2014, two sea lamprey (1.4\%), both from group 3 , had lost their tags. Incision health on March 4, 2014, varied from healed skin to large muscle bulges near the incision (Table 3). Of the lamprey that had not lost their

Table 1 Mean total length, percent survival, and percent retention of 12-mm PIT tags after 41-118 day for metamorphosing juvenile sea lamprey from the Fort River, MA and two Lake Champlain tributaries

\begin{tabular}{|c|c|c|c|c|c|c|c|c|c|}
\hline \multirow[t]{2}{*}{ Source/group } & \multirow[t]{2}{*}{$n$} & \multirow{2}{*}{$\begin{array}{l}\text { Tagging/monitoring } \\
\text { date }\end{array}$} & \multirow{2}{*}{$\begin{array}{l}\text { Total days } \\
\text { monitored }\end{array}$} & \multicolumn{2}{|c|}{ Total length (mm) } & \multicolumn{2}{|c|}{ Survival (\%) } & \multicolumn{2}{|c|}{ Tag retention (\%) } \\
\hline & & & & Mean & Range & $24 \mathrm{~h}$ & Overall & $24 \mathrm{~h}$ & $\begin{array}{l}\text { Overall (\# of days } \\
\text { tagged) }\end{array}$ \\
\hline \multicolumn{10}{|l|}{ Untagged } \\
\hline Fort River Group 1 & 23 & November 19, 2013 & 64 & 142.9 & $125-167$ & 100 & 100.0 & $\mathrm{~N} / \mathrm{A}$ & $\mathrm{N} / \mathrm{A}$ \\
\hline Fort River Group 2 & 15 & December 6, 2013 & 47 & 145.5 & $134-156$ & 100 & 100.0 & N/A & N/A \\
\hline Lake Champlain & 120 & December 6, 2013 & 60 & * & $*$ & 100 & 94.2 & N/A & $\mathrm{N} / \mathrm{A}$ \\
\hline \multicolumn{10}{|l|}{ Tagged } \\
\hline Fort River Group 1 & 20 & November 19, 2013 & 118 & 147.4 & $129-176$ & 100 & 90.0 & 100 & $100.0(105)$ \\
\hline Fort River Group 2 & 20 & December 6, 2013 & 101 & 148.0 & $135-168$ & 100 & 85.0 & 100 & $100.0(88)$ \\
\hline Lake Champlain & 110 & February 4, 2014 & 41 & 167.8 & $139-205$ & 100 & 94.5 & 100 & $98.2(28)$ \\
\hline Total tagged & 150 & $\mathrm{~N} / \mathrm{A}$ & $\mathrm{N} / \mathrm{A}$ & 162.4 & $129-205$ & 100 & 92.7 & 100 & $98.6(-)$ \\
\hline
\end{tabular}

Percent tag retention of all surviving lamprey was evaluated on March 4, 2014. Lake Champlain tagged and untagged sea lamprey consisted of one group monitored for 60 day pre-tagging $\left.{ }^{*}\right)$, then tagged and monitored for 41 day post-tagging 

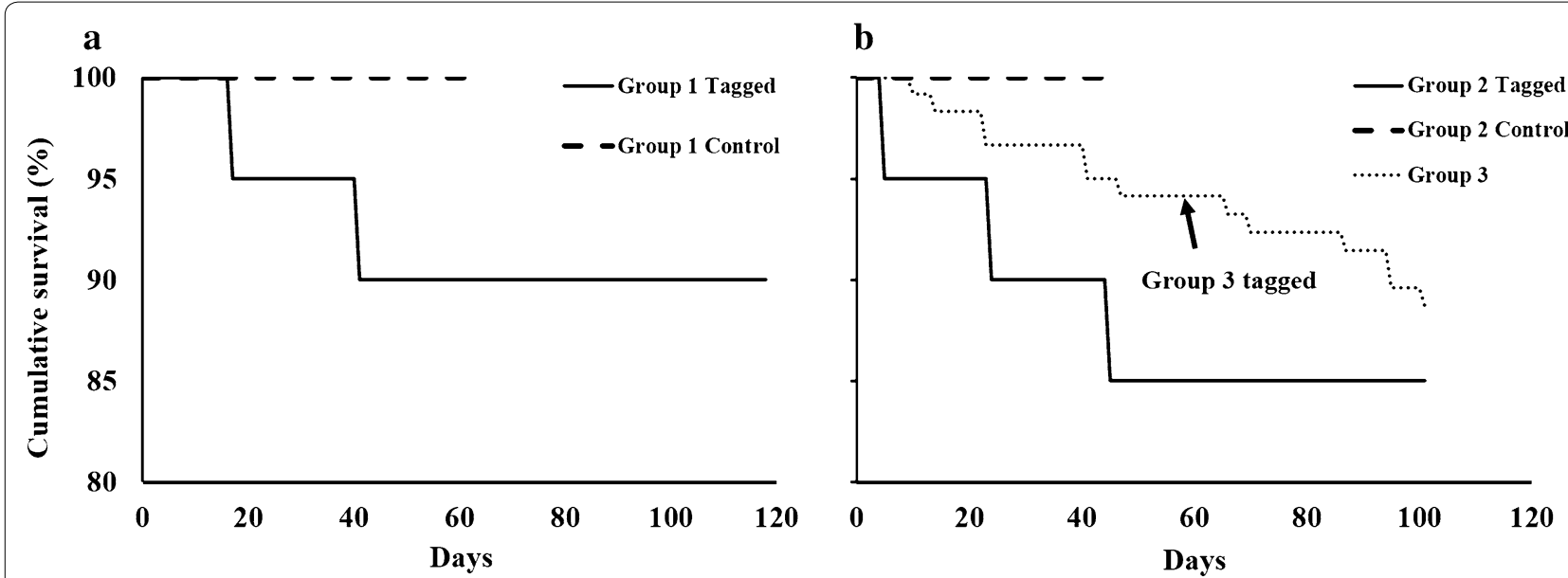

Fig. 2 Percent cumulative survival for three groups of sea lamprey implanted with 12-mm HDX PIT tags and held in laboratory tanks. Group 1 (a) and group 2 (b) sea lamprey were collected from Fort River, MA, tagged (solid lines) and held in observation tanks with untagged controls (dashed lines), and monitored for 64 and $47 \mathrm{~d}$, respectively. Group 1 and 2 tagged sea lamprey were held for an additional 54 day after control animals were removed to continue monitoring survival and tag retention. Group 3 sea lamprey (b, dotted gray line) were collected from two tributaries to Lake Champlain and monitored for $60 \mathrm{~d}$; they were then tagged and monitored for 41 day post-tagging to assess survival and tag retention

Table 3 Percentage of incision conditions observed for metamorphosing juvenile sea lamprey from three groups surgically implanted with 12-mm HDX PIT tags

\begin{tabular}{lccc}
\hline Incision condition & Fort River group $\mathbf{1}(\boldsymbol{n = 1 8 , 1 0 5} \mathbf{d})$ & Fort River group $\mathbf{2}(\boldsymbol{n}=\mathbf{1 7 , 8 8} \mathbf{d})$ & $\begin{array}{l}\text { Lake Champlain } \\
(\boldsymbol{n}=\mathbf{1 0 7 , 2 8} \mathbf{d})\end{array}$ \\
\hline Skin healed & & & $14.0(15)$ \\
Internal muscle healed & $72.2(13)$ & $17.6(3)$ & $51.4(55)$ \\
Internal muscle closed & $27.8(5)$ & $47.1(8)$ & $21.5(23)$ \\
Subtotal & $0.0(0)$ & $11.8(2)$ & $86.9(93)$ \\
Status not determined, no muscle bulge & $100.0(18)$ & $76.5(13)$ & $1.9(2)$ \\
Open, no muscle bulge & $0.0(0)$ & $0.0(0)$ & $0.9(1)$ \\
Small muscle bulge & $0.0(0)$ & $0.0(0)$ & $7.5(8)$ \\
Medium muscle bulge & $0.0(0)$ & $23.5(4)$ & $0.9(1)$ \\
Large muscle bulge & $0.0(0)$ & $0.0(0)$ & $1.9(2)$ \\
Subtotal & $0.0(0)$ & $0.0(0)$ & $13.1(14)$ \\
\hline
\end{tabular}

Sea lamprey were evaluated after 28-105 d; sample sizes are given in parentheses. Only surviving sea lamprey that had not shed their PIT tags were evaluated

tag, $87.3 \%$ had incisions classified as 'skin healed,' 'internal muscle healed,' or 'internal muscle closed' and were no longer at risk of tag loss. The remaining lamprey had open incisions, either with or without protruding muscle bulges, and were considered to be still at risk of tag loss.

\section{Discussion}

Our results demonstrate that metamorphosing juvenile sea lamprey can be tagged with 12-mm PIT tags using a field-amenable, simple tagging method [20], and exhibit high survival and tag retention. The sea lamprey used in this experiment were collected at different times, sourced from three different streams, held for different lengths of time before and after tagging, and varied widely in length, yet overall survival at the end of an extended observation period was nearly $93 \%$. By demonstrating that survival and tag retention can be high regardless of these different conditions, our results are applicable to a wide range of future studies in which these conditions are likely to vary as well.

Survival in our experiment was similar to that seen in other experiments where outmigrating juvenile Pacific Lamprey were tagged and held in cool water [17, 19, 20]. Tagged Pacific Lamprey held at 8 and $9{ }^{\circ} \mathrm{C}$ exhibited slightly lower overall mortality than we observed at $5.2{ }^{\circ} \mathrm{C}$; however, this difference was minimal when 
compared with the dramatic increase in mortality observed for Pacific Lamprey held at temperatures between 12 and $23{ }^{\circ} \mathrm{C}[19,20]$. Most mortalities observed during other experiments with tagged juvenile lampreys resulted from fungal infection, which appeared to be a severe issue in several cases [17-20]. Only one tagged and three untagged sea lamprey died in our experiment as a result of fungus. We did not treat sea lamprey to inhibit fungal growth in this experiment and assumed fungal infections were a product of captive conditions. If tagging does not affect the rate of fungal infection in a natural environment, lamprey could be released $24 \mathrm{~h}$ after tagging to reduce this source of mortality. Further studies should investigate survival of lamprey released immediately after tagging, which would reduce stresses due to handling, transportation, and captivity.

A greater proportion (3 of 8; 38\%) of tagged lamprey $<140 \mathrm{~mm}$ died during our experiment than larger lamprey (8 of 142; 6\%). Though our sample of $<140 \mathrm{~mm}$ lamprey was small, other lamprey tagging studies have suggested thresholds similar to this value. Mueller et al. [19] also used 12-mm tags in juvenile Pacific Lamprey and recommended a threshold of $120 \mathrm{~mm}$; however, their surgical process utilized a needle to inject the tag through a small puncture in the skin that was then closed by a single suture. Although this process appears to allow smaller individuals to be tagged, the process may be too complicated for use in the field and a $135-\mathrm{mm}$ threshold was suggested when following the simpler tagging procedure to implant 9-mm tags [20]. Low survival was observed for larval sea lamprey with an average length under $120 \mathrm{~mm}$ even with 8-mm tags (40\% survival) and 9-mm tags (10\% survival; [22]); however, recent research demonstrated substantially higher survival rates (97\% field; 94\% laboratory) for larval Pacific Lamprey implanted with $8.4 \mathrm{~mm} \times 1.4 \mathrm{~mm}$ FDX PIT tags [23]. Schreck et al. [18] suggested $150 \mathrm{~mm}$ as a minimum size using $8-\mathrm{mm}$ PIT tags; our findings suggest this may be conservative as $82.3 \%$ of lamprey in our study $<150 \mathrm{~mm}$ survived with larger tags $(12 \mathrm{~mm} \times 2.12 \mathrm{~mm})$.

Demonstrating high survival for juvenile sea lamprey implanted with the 12-mm HDX tag provides managers and researchers with a tool for understanding downstream migration behavior of sea lamprey where monitoring larger open channels is required. Previous field studies of PIT tagged lamprey have been limited to the detection of ammocoetes or lamprey that had begun to metamorphose using portable detection equipment that can be slowly and repeatedly passed over an area [17, 22]. Although shorter lamprey can be successfully tagged with 8.4-mm PIT tags [23], detection range is generally reduced as tag size decreases [12]. To effectively detect outmigrating sea lamprey, studies should utilize the largest tags possible to maximize the probability of detection when using stream-spanning wire arrays. Antenna arrays for larger HDX tags also do not require the rigid, watertight enclosures necessary for the FDX tags allowing for easier installation at a lower cost.

PIT telemetry has proved to be a valuable tool for management of downstream migrating salmon smolts and has provided a wealth of information about this critical early-life period, much of which remains unknown for sea lamprey. For example, PIT telemetry has been used to determine system-wide, reach-specific, and seasonal survival [24, 25], temporal and spatial movement patterns [26], and timing of movement and survival relative to stream conditions and hydropower operations [27, 28]. Ultimately, having the capability to monitor downstream movement in natural, open, stream channels can provide a better understanding of when and how sea lamprey outmigrate. This tool will allow managers to understand where and when invasive sea lamprey outmigrants in the Great Lakes are most vulnerable to capture and assess risk of mortality during outmigration for sea lamprey in their native range.

\section{Conclusions}

Demonstrating the use of larger HDX tags as a viable option for PIT telemetry for these small fish provides another monitoring tool for researchers who might find the FDX PIT systems too costly or technically prohibitive but wish to maximize detection range. Given that juvenile sea lamprey typically migrate during periods of high flow and often heavy debris load (leaves, snow, ice), antenna arrays can be damaged or completely destroyed. A cheaper alternative for antennas allows monitoring in open stream channels where equipment damage is more likely.

The overall high level of survival and tag retention in this study indicates that future studies can successfully use metamorphosing juvenile sea lamprey implanted with $12-\mathrm{mm}$ HDX PIT tags to better understand their downstream movement patterns and timing of outmigration. Currently in the Great Lakes, no control effort targets the downstream migratory phase, in large part due to the protracted migratory period. Understanding when downstream movement occurs and how this movement relates to environmental cues would allow managers to target their effort during periods of higher likelihood of capture to maximize trapping benefits relative to cost of operations.

\footnotetext{
Authors' contributions

SM conceived the work on metamorphosing sea lamprey and advised throughout the work; LGS and VAS conducted the sampling, tagging, culture, and tag retention experiments and analyzed the data; LGS prepared the manuscript with assistance with JEM and SM. All authors read and approved the final manuscript.

\section{Author details}

${ }^{1}$ Rubenstein Ecosystem Science Laboratory, University of Vermont, 3 College St., Burlington, VT 05401, USA. ${ }^{2}$ U.S. Geological Survey, Great Lakes Science
} 
Center, Hammond Bay Biological Station, 11188 Ray Road, Millersburg, MI 49759, USA.

\section{Acknowledgements}

We thank the Vermont Fish and Wildlife Department and the US Fish and Wildlife Service for support with sampling locations and the Conte Anadromous Fish Research Laboratory for providing lamprey from the Fort River. Bret Ladago, Harrison Tobi, Elizabeth Puchala, and Bill Lamoreux provided field assistance with lamprey collections in Lake Champlain tributaries. Any use of trade names is for descriptive purposes only and does not imply endorsement by the US Government.

\section{Competing interests}

The authors declare that they have no competing interests.

\section{Availability of data and materials}

The datasets used and/or analyzed during the current study are available from the corresponding author on reasonable request.

\section{Ethics approval}

Fish care and protocols for fish holding, surgery, and tagging were conducted under a University of Vermont Institutional Animal Care and Use Committee Permit 13-017.

\section{Funding}

This work was funded by the Great Lakes Fishery Commission.

\section{Publisher's Note}

Springer Nature remains neutral with regard to jurisdictional claims in published maps and institutional affiliations.

Received: 12 March 2017 Accepted: 2 July 2017

Published online: 14 July 2017

\section{References}

1. Applegate VC. The sea lamprey in the Great Lakes. Sci Month. 1951;72:275-81.

2. Smith BR, Tibbles JJ. Sea lamprey (Petromyzon marinus) in lakes Huron, Michigan, and Superior-history of invasion and control, 1936-78. Can J Fish Aquat Sci. 1980;37:1780-801.

3. Lavis DS, Hallett A, Koon EM, McAuley TC. History of and advances in barriers as an alternative method to suppress sea lampreys in the Great Lakes. J Great Lakes Res. 2003;29(Suppl 1):362-72.

4. Hunn J, Youngs W. Role of physical barriers in the control of sea lamprey (Petromyzon marinus). Can J Fish Aquat Sci. 1980;37:2118-22.

5. Twohey MB, Heinrich JW, Seelye JG, Fredricks KT, Bergstedt RA, Kaye CA, Scholefield RJ, McDonald RB, Christie GC. The sterile-male-release technique in Great Lakes sea lamprey management. J Great Lakes Res. 2003;29(Supplement 1):410-23.

6. Applegate VC, Brynildson CL. Downstream movement of recently transformed sea lampreys, Petromyzon marinus, in the Carp Lake River, Michigan. Trans Am Fish Soc. 1952;81:275-90.

7. Smith JW, Swink WD. Boll weevil eradication: a model for sea lamprey control? J Great Lakes Res. 2003;29(Suppl 1):445-55.

8. Connor WP, Burge HL, Bennett DH. Detection of PIT-tagged subyearling Chinook Salmon at a Snake River Dam: implications for summer flow augmentation. N Am J Fish Manag. 1998;18:530-6.

9. Pratt TC, O'Connor LM, Hallett AG, McLaughlin RL, Katopodis C, Hayes DB, Bergstedt RA. Balancing aquatic habitat fragmentation and control of invasive species: enhancing selective fish passage at sea lamprey control barriers. Trans Am Fish Soc. 2009;138:652-65.
10. Benjamin JR, Wetzel LA, Martens KD, Larsen K, Connolly PJ, Gillanders B. Spatio-temporal variability in movement, age, and growth of mountain whitefish (Prosopium williamsoni) in a river network based upon PIT tagging and otolith chemistry. Can J Fish Aquat Sci. 2014;71:131-40.

11. Gibbons JW, Andrews KM. PIT tagging: simple technology at its best. Bioscience. 2004;54:447-54

12. Zydlewski GB, Horton G, Dubreuil T, Letcher B, Casey S, Zydlewski J. Remote monitoring of fish in small streams. Fisheries. 2006;31:492-502.

13. Castro-Santos T, Haro A, Walk S. A passive integrated transponder (PIT) tag system for monitoring fishways. Fish Res. 1996;28:253-61.

14. Morhardt JE, Bishir D, Handlin CI, Mulder SD. A portable system for reading large passive integrated transponder tags from wild trout. N Am J Fish Manag. 2000;20:276-83.

15. Zydlewski GB, Haro A, Whalen K, McCormick S. Performance of stationary and portable passive transponder detection systems for monitoring of fish movements. J Fish Biol. 2001:58:1471-5.

16. Ibbotson A, Beaumont W, Collinson D, Wilkinson A, Pinder A. A cross-river antenna array for the detection of miniature passive integrated transponder tags in deep, fast flowing rivers. J Fish Biol. 2004;65:1441-3.

17. Quintella BR, Andrade NO, Espanhol R, Almeida PR. The use of PIT telemetry to study movements of ammocoetes and metamorphosing sea lampreys in river beds. J Fish Biol. 2005;66:97-106.

18. Schreck C, Fitzpatrick M, Lerner D. Determination of passage of juvenile lamprey: development of a tagging protocol. Oregon State University, Oregon Cooperative Fish and Wildlife Research Unit, Corvalis; 1999.

19. Mueller RP, Moursund RA, Bleich MD. Tagging juvenile Pacific lamprey with passive integrated transponders: methodology, short-term mortality, and influence on swimming performance. N Am J Fish Manag. 2006:26:361-6.

20. Mesa MG, Copeland ES, Christiansen HE, Gregg JL, Roon SR, Hershberger PK. Survival and growth of juvenile Pacific lampreys tagged with passive integrated transponders (PIT) in freshwater and seawater. Trans Am Fish Soc. 2012;141:1260-8.

21. Slade JW, Adams JV, Christie GC, Cuddy DW, Fodale MF, Heinrich JW, Quinlan HR, Weise JG, Weisser JW, Young RJ. Techniques and methods of estimating abundance of larval and metamorphosed sea lampreys in Great Lakes tributaries, 1995 to 2001. J Great Lakes Res. 2003;29(Supplement 1):137-51.

22. Dawson HA, Potts DD, Maguffee AC, O'Connor LM. Feasibility of passive integrated transponder technology to study in situ movements of larval sea lamprey. J Fish Wildl Manag. 2015;6:71-82.

23. Moser ML, Jackson AD, Mueller RP, Maine AN, Davisson M. Effects of passive integrated transponder (PIT) implantation on Pacific lamprey ammocoetes. Anim Biotelemetry. 2017:5:1.

24. Skalski JR. Estimating season-wide survival rates of outmigrating salmon smolt in the Snake River, Washington. Can J Fish Aquat Sci. 1998:55:761-9.

25. Skalski JR, Smith SG, Iwamoto RN, Williams JG, Hoffmann A. Use of passive integrated transponder tags to estimate survival of migrant juvenile salmonids in the Snake and Columbia rivers. Can J Fish Aquat Sci. 1998:55:1484-93.

26. Homel K, Budy P. Temporal and spatial variability in the migration patterns of juvenile and subadult Bull Trout in northeastern Oregon. Trans Am Fish Soc. 2008:137:869-80.

27. Budy P, Thiede GP, Bouwes N, Petrosky CE, Schaller H. Evidence linking delayed mortality of Snake River salmon to their earlier hydrosystem experience. N Am J Fish Manag. 2002;22:35-51.

28. Skalski JR, Eppard MB, Ploskey GR, Weiland MA, Carlson TJ, Townsend RL. Assessment of subyearling Chinook Salmon survival through the federal hydropower projects in the main-stem Columbia River. N Am J Fish Manag. 2014;34:741-52. 